\title{
Efecto del Campo Magnético sobre la Rectificación Óptica en un Punto Cuántico Cilíndrico
}

\author{
Alfonso A. Portacio(1)* Andrés F. Jiménez $^{(1)}$ y María del Pilar Urango ${ }^{(2)}$ \\ (1) Universidad de los Llanos, Departamento de Matemáticas y Física, Facultad de Ciencias Básicas e \\ Ingenierías, Km 12 vía puerto López, Villavicencio - Meta, Colombia. (e-mail: aportacio@unillanos.edu.co) \\ (2) Universidad de los Llanos, Departamento de Biología y Química, Facultad de Ciencias Básicas e \\ Ingenierías, Km 12 vía puerto López, Villavicencio - Meta, Colombia.
}

${ }^{*}$ Autor a quien debe dirigirse la correspondencia.

Recibido Abr. 4, 2016; Aceptado May. 30, 2016; Versión final Jun. 22, 2016, Publicado Dic. 2016

\begin{abstract}
Resumen
Se estudia teóricamente la rectificación óptica no lineal en un punto cuántico cilíndrico (CQD) en presencia de un campo magnético uniforme usando el formalismo de la matriz densidad y un procedimiento perturbativo. Los niveles de energía y las funciones de onda de un electrón en el CQD se obtuvieron solucionando la ecuación de Schrödinger independiente del tiempo en el marco de la aproximación de masa efectiva. Los cálculos numéricos se efectúan para un CQD del tipo GaAs/AIGaAs. Se encontró que el aumento en las dimensiones del CQD y/o la intensidad del campo magnético causan un aumento en su intensidad sobre el máximo de resonancia y un desplazamiento hacia regiones de energías bajas (corrimiento al rojo). La razón física de este comportamiento se atribuye al efecto del confinamiento cuántico generado por la variación de la intensidad del campo magnético y de las dimensiones de la nanoestructura.
\end{abstract}

Palabras clave: rectificación óptica, punto cuántico cilíndrico, campo magnético, matriz densidad

\section{Effects of Magnetic Field on the Optical Rectification in a Cylindrical Quantum Dot}

\begin{abstract}
A theoretical study on the nonlinear optical rectification in a cylindrical quantum dot (CQD) in the presence of a uniform magnetic field by using the density matrix formalism and a perturbation method is presented. The energy levels and wave functions of an electron in the CQD were obtained by solving the timeindependent Schrödinger equation within the effective mass approximation. Numerical calculations are performed for a CQD of the type GaAs/AIGaAs. It was found that the increase in the dimensions of CQD and/or the magnetic field intensity cause an increase on the resonance peak intensity and a shift to lower energies regions (red shift). The physical reason for this behavior is attributed to the quantum confinement effect generated by the variation of the magnetic field strength and of the size of the nanostructure.
\end{abstract}

Keywords: optical rectification, cylindrical quantum dot, magnetic field, density matrix 


\section{INTRODUCCIÓN}

El desarrollo de las técnicas de crecimiento y de caracterización de materiales han permitido diseñar y producir una gran variedad de sistemas cuánticos de baja dimensionalidad que incluyen pozos cuánticos, hilos cuánticos y puntos cuánticos (QD's - del inglés) (Rezaei et al., 2014), que han contribuido con la evolución de la nano-electrónica y el desarrollo de tecnologías tales como: producción de láseres, fuentes de emisión de fotones, relojes cuánticos y algunas tecnologías adecuadas para información cuántica, computación cuántica, etc. (Vaseghi et al., 2015). Diferentes investigaciones afirman que el confinamiento cuántico produce una respuesta óptica no lineal más notoria en sistemas nano-estructurados que en los materiales en bloque (Wang., 2015; Sakar et al., 2016). Este hecho ha despertado el interés de investigadores, teóricos y/o experimentales y por ello se encuentran reportados diversos estudios sobre la respuesta óptica no lineal en sistemas de baja dimensionalidad, donde los QD's son las nano-estructuras más exploradas porque el confinamiento tridimensional de los electrones en estas estructuras conduce a la formación de espectros electrónicos de energía similares a los de sistemas atómicos; estos niveles discretos de energía se pueden ajustar mediante el control del tamaño de los QD's y la aplicación de campos externos (Hoyos et al., 2016). Estas propiedades hacen a los QD's candidatos atractivos para diseñar dispositivos opto-electrónicos de alto rendimiento (Rezaei et al., 2014; Akimoto et al., 2015).

Las propiedades ópticas no lineales de segundo orden incluidas la rectificación óptica (OR - del inglés) y la generación de segundo armónico, son los efectos ópticos no lineales de orden más bajo que poseen una magnitud generalmente mayor que los fenómenos ópticos no lineales de orden superior; siempre que el sistema cuántico muestre cierta asimetría (Xie., 2014). Este tipo de asimetría puede ser producida mediante el uso de tecnología avanzada de crecimiento de materiales, que permite obtener nano-estructuras con un potencial de confinamiento asimétrico y también por la aplicación de un campo eléctrico estático a un sistema estructuralmente simétrico. (Abdullah et al., 2015; Li et al., 2014). La OR y/o susceptibilidades de segundo orden han sido investigadas en varios tipos de QD's, tales como: sistemas cuánticos parabólicos (Shojaei et al., 2015), sistemas cuánticos esféricos (Vaseghi et al., 2015), sistemas cuánticos cúbicos (Zhang et al., 2009) entre otros. Sin embargo se ha reportado que, la magnitud del coeficiente de OR en puntos cuánticos cilíndricos (CQD) es mayor que en los sistemas de puntos cuánticos esféricos, pero se encuentran pocos trabajos sobre OR en sistemas con confinamiento cilíndrico ( $\mathrm{Li}$ et al., 2014). Así que investigaciones sobre OR en CQD son importantes para aplicaciones teóricas y/o prácticas.

Por otra parte, los campos externos se han convertido en un problema interesante para el estudio de las propiedades físicas de los sistemas cuánticos de baja dimensionalidad, tanto desde el punto de vista teórico como experimental. Shojaei et al., estudiaron el rol del campo magnético sobre la OR no lineal de una impureza hidrogenoide en un punto cuántico parabólico en forma de disco, mostrando que el campo magnético cambia los niveles de energía y por ende la respuesta óptica (Shojaei et al., 2015). Duque et al., estudiaron el efecto de un láser intenso sobre la absorción óptica no lineal y OR en pozos cuánticos individuales sometidos a un campo eléctrico y magnético, mostrando que la absorción óptica no lineal y rectificación óptica son funciones no monótonas de las dimensiones de la heteroestructura y de las perturbaciones externas que consideraron en su investigación (Duque et al., 2011). Esta investigación presenta un estudio teórico sobre la rectificación óptica en un CQD de GaAs/AIGaAs con potencial de confinamiento asimétrico, sometido a un campo magnético uniforme. Se analiza el efecto del campo magnético y del tamaño del CQD.

\section{TEORÍA}

El sistema cuántico estudiado consiste de un electrón confinado en CQD en presencia de un campo magnético uniforme $B$ orientado en la dirección axial del CQD. En el marco de la aproximación de masa efectiva el Hamiltoniano $\widehat{H}_{0}$ del sistema está dado por (Villamil., 2010; Liu et al., 2012):

$\widehat{H}_{0}=\frac{1}{2 m^{*}}\left(\widehat{\boldsymbol{P}}-\frac{e}{c} \boldsymbol{A}\right)^{2}+V_{\text {conf }}(\boldsymbol{r})$,

donde $m^{*}$ es la masa efectiva, $\widehat{\boldsymbol{P}}$ es el operador momento lineal, $e$ es la carga fundamental, $c$ es la velocidad de la luz en el vacío, $A$ es el vector potencial del campo magnético uniforme, el cual se puede escribir como $A(r)=1 / 2 B \times r$ con $B=B z$ y en coordenadas cilíndricas se convierte en $A_{\rho}=A_{z}=0, A_{\varphi}=1 / 2 B \rho . V_{\text {conf }}(r)$, es un potencial de confinamiento finito, el cual incluye potenciales parabólicos tanto en dirección radial como a lo largo de z (Khordad., 2013; Li et al., 2014) y un término asimétrico en la coordenada z; como se muestra a continuación:

$$
V_{\text {conf }}(\boldsymbol{r})=V_{\text {conf }}(\rho, \varphi, z)=\frac{m^{*} \omega_{\rho}^{2} \rho^{2}}{2}+\frac{m^{*} \omega_{Z}^{2} z^{2}}{2}+\alpha \frac{m^{*} \omega_{Z}^{2} z^{3}}{2 L}
$$


donde $\omega_{\rho} \sim \frac{\hbar}{m^{*} R^{2}}$ y $\omega_{z} \sim \frac{\hbar}{m^{*} L^{2}}$, con $\mathrm{R}$ y $\mathrm{L}$ el radio y la longitud del cilindro respectivamente, $\alpha$ es un parámetro adimensional que indica la intensidad de la perturbación asimétrica. La ecuación de Schrödinger en coordenadas cilíndricas tiene la forma:

$$
-\frac{\hbar^{2}}{2 m^{*}}\left[\frac{1}{\rho} \frac{\partial}{\partial \rho}\left(\rho \frac{\partial}{\partial \rho}\right)+\frac{1}{\rho^{2}} \frac{\partial^{2}}{\partial \varphi^{2}}+\frac{\partial^{2}}{\partial z^{2}}\right] \psi-\frac{i \hbar \omega_{c}}{2} \frac{\partial \psi}{\partial \varphi}+\frac{m^{*} \omega_{c}^{2} \rho^{2}}{8} \psi+\frac{m^{*} \omega_{\rho}^{2} \rho^{2}}{2} \psi+\frac{m^{*} \omega_{Z}^{2} z^{2}}{2} \psi+\alpha \frac{m^{*} \omega_{Z}^{2} z^{3}}{2 L} \psi=E \psi,
$$

donde $\omega_{c}=\frac{e B}{m^{*} c}$ es la frecuencia de ciclotrón. Se propone el siguiente ansatz para la función de onda del electrón:

$\psi(\rho, \varphi, z)=f(\rho, \varphi) g(z)$.

Al insertar (4) en (3) se obtienen una ecuación para $f(\rho, \varphi)$ y otra para $g(z)$, esto es:

$-\frac{\hbar^{2}}{2 m^{*}}\left[\frac{1}{\rho} \frac{\partial}{\partial \rho}\left(\rho \frac{\partial f}{\partial \rho}\right)+\frac{1}{\rho^{2}} \frac{\partial^{2} f}{\partial \varphi^{2}}\right]-\frac{i \hbar \omega_{c}}{2} \frac{\partial f}{\partial \varphi}+\frac{m^{*} \Omega^{2} \rho^{2}}{8} f=E_{\rho} f$,

donde $\Omega=\sqrt{\omega_{c}^{2}+4 \omega_{\rho}^{2}}$ y

$-\frac{\hbar^{2}}{2 m^{*}} \frac{d^{2} g}{d z^{2}}+\frac{m^{*} \omega_{Z}^{2} Z^{2}}{2} g+\alpha \frac{m^{*} \omega_{Z}^{2} Z^{3}}{2 L} g=E_{z} g$.

La solución de la ecuación (5) es conocida (Li et al., 2014) y está dada por:

$f_{m, n_{\rho}}(\rho, \varphi)=\frac{1}{\sqrt{2 \pi}} \frac{1}{a^{1+|m|}}\left[\frac{\left(|m|+n_{\rho}\right) !}{2|m| n_{\rho} !|m| !}\right]^{\frac{1}{2}} e^{i m \varphi} e^{-\frac{\rho^{2}}{4 a^{2}}} \rho^{|m|} F\left(-n_{\rho},|m|+1, \frac{\rho^{2}}{2 a^{2}}\right)$,

donde $a=\sqrt{\hbar / m^{*} \Omega}, F(a, b, x)$ es una función hipergeométrica confluente, $m$ es el número cuántico magnético y $n_{\rho}$ es el número cuántico radial. La ecuación (6) se resuelve usando teoría de perturbaciones independiente del tiempo, donde el término $\alpha \frac{m^{*} \omega_{Z}^{2} z^{3}}{2 L}$ corresponde a la perturbación y el problema sin perturbar corresponde a un oscilador armónico unidimensional cuya solución es conocida (Khordad., 2013) y está dada por:

$g_{n}^{(0)}(z)=\left(\frac{m^{*} \omega_{z}}{\pi \hbar}\right)^{\frac{1}{4}} \frac{1}{\sqrt{2^{n} n !}} e^{-\frac{m^{*} \omega_{Z} z^{2}}{\hbar}} H_{n}\left(\sqrt{\frac{m^{*} \omega_{Z}}{2 \hbar}} z\right)$,

$E_{z}^{(0)}=\hbar \omega_{z}\left(n+\frac{1}{2}\right)$

donde, $H_{n}(x)$ son los polinomios de Hermite y $n$ es un número cuántico. La corrección de la función de onda y la energía después de aplicar la teoría de perturbaciones independiente del tiempo son:

$$
\begin{aligned}
& g_{n}(z)=g_{n}^{(0)}(z)+\frac{\alpha m^{*} \omega_{z}}{2 L \hbar}\left(\frac{\hbar}{2 m^{*} \omega_{z}}\right)^{\frac{3}{2}} \\
& \left(-\frac{\sqrt{(n+1)(n+2)(n+3)}}{3} g_{n+3}^{(0)}(z)+\frac{\sqrt{n(n-1)(n-2)}}{3} g_{n-3}^{(0)}(z)-3(n+1)^{\frac{3}{2}} g_{n+1}^{(0)}(z)+3 n^{\frac{3}{2}} g_{n-1}^{(0)}(z)\right) \\
& E_{z}=\hbar \omega_{z}\left(n+\frac{1}{2}\right)-\frac{(\alpha \hbar)^{2}}{4 m^{*} L^{2}}\left[\frac{15}{4}\left(n+\frac{1}{2}\right)^{2}+\frac{7}{16}\right] .
\end{aligned}
$$

La fórmula para rectificación óptica se derivó usando el método de matriz densidad y su procedimiento perturbativo, para ello se consideró la interacción del sistema cuántico con un campo óptico de luz de la forma $E(t)=\widetilde{E} e^{-i \omega t}+\widetilde{E}^{*} e^{i \omega t}$, la evolución del operador matriz densidad del sistema $\hat{\rho}(t)$ está dada por la siguiente ecuación maestra fenomenológica (Rosencher et al., 1991; Vaseghi et al., 2015).

$\frac{\partial \widehat{\rho}}{\partial t}=\frac{1}{i \hbar}\left[\widehat{H}_{0}+\widehat{V}(t), \hat{\rho}(t)\right]-\hat{\Gamma}\left(\hat{\rho}(t)-\hat{\rho}^{(0)}(t)\right)$,

donde, $\widehat{H}_{0}$ representa el operador Hamiltoniano del sistema cuántico sin interactuar con el campo óptico, $\widehat{V}(t)=-\hat{\mu} \cdot E(t)$ es el operador Hamiltoniano de interacción entre el campo óptico $E(t)$ y la nano-estructura 
en la aproximación dipolar, $\hat{\mu}$ es el operador momento dipolar, $\hat{\rho}^{(0)}(t)$ es el operador densidad del sistema no perturbado, $\hat{\Gamma}$ es el operador fenomenológico responsable de los procesos de relajación y sus elementos matriciales se asocian con los tiempos de relajación de los procesos de emisión espontánea y pérdida de coherencia del sistema.

Para resolver (12) se utiliza el procedimiento perturbativo estándar que implica la expansión de $\hat{\rho}(t)$ :

$$
\hat{\rho}(t)=\sum_{i} \hat{\rho}^{(i)}(t) .
$$

El conocimiento de la contribución de orden $i$ del operador matriz densidad $\hat{\rho}^{(i)}$ permite calcular la polarización electrónica de orden $n$ mediante la siguiente ecuación:

$\left\langle P^{(i)}(t)\right\rangle=\frac{1}{V} \operatorname{Tr}\left[\hat{\rho}^{(i)} \hat{\mu}\right]$,

donde, $V$ es el volumen del punto cuántico. Por otra parte, según la electrodinámica clásica la polarización eléctrica inducida $P(t)$ en el CQD debido a la interacción con el campo óptico $E(t)$ se escribe como:

$P(t)=\varepsilon_{0}\left(\chi^{(1)} \tilde{E} e^{-i \omega t}+\chi_{0}^{(2)}|\tilde{E}|^{2}+\chi_{2 \omega}^{(2)} \tilde{E}^{2} e^{-i 2 \omega t}+\chi_{3 \omega}^{(3)} \tilde{E}^{3} e^{-i 3 \omega t}\right)+$ c.c.,

donde, $\chi^{(1)}, \chi_{0}^{(2)}, \chi_{2 \omega}^{(2)}, \chi_{3 \omega}^{(3)}$ son la susceptibilidad óptica lineal, la rectificación óptica, generación de segundo armónico y generación de tercer armónico respectivamente y $\varepsilon_{0}$ es la permitividad del vacío.

La expresión analítica para el coeficiente de rectificación óptica por unidad de volumen se obtiene a partir de las ecuaciones (14) y (15) y está dada por:

$\chi_{0}^{(2)}=\frac{4 \sigma_{v}}{\varepsilon_{0}} M_{f i}^{2} \delta_{f i} \frac{E_{f i}^{2}\left(1+\frac{T_{1}}{T_{2}}\right)+\left((\hbar \omega)^{2}+\frac{\hbar}{T_{2}^{2}}\right)\left(\frac{T_{1}}{T_{2}}-1\right)}{\left[\left(E_{f i}-\hbar \omega\right)^{2}+\left(\frac{\hbar}{T_{2}}\right)^{2}\right]\left[\left(E_{f i}+\hbar \omega\right)^{2}+\left(\frac{\hbar}{T_{2}}\right)^{2}\right]}$,

donde $\sigma_{v}$ es la densidad de electrones en el CQD, $E_{f i}$ es la energía de transición del estado $\psi_{i}$ (con $\left.\mathrm{n}_{\rho}=\mathrm{m}=\mathrm{n}=0\right)$ al estado $\psi_{f}\left(\right.$ con $\left.\mathrm{m}=0 ; \mathrm{n}_{\rho}=\mathrm{n}=1\right), M_{f i}=\left|\left\langle\psi_{f}|e z| \psi_{i}\right\rangle\right|$ es el momento dipolar eléctrico de transición del estado $\psi_{i}$ al estado $\psi_{f}, \delta_{f i}=\left|M_{f f}-M_{i i}\right|, \hbar \omega$ es la energía del fotón incidente, $\mathrm{T}_{1}$ y $\mathrm{T}_{2}$ son los tiempos de relajación del sistema longitudinal y transversal respectivamente (West., et al 1985; Zhang et al., 2009).

\section{RESULTADOS Y DISCUSIÓN}

En esta sección, se discute el coeficiente de rectificación óptica de CQD de GaAs/AIGaAs bajo la influencia de un campo magnético externo. En estos cálculos se usaron los siguientes parámetros: $m^{*}=0.067 m_{0}\left(m_{0}\right.$ es la masa del electrón libre) $T_{1}=1 \mathrm{ps}, \mathrm{T}_{2}=0.14 \mathrm{ps}, \sigma_{\mathrm{v}}=5 \times 10^{24} \mathrm{~m}^{-3}$ (West., et al 1985; Li et al., 2014).

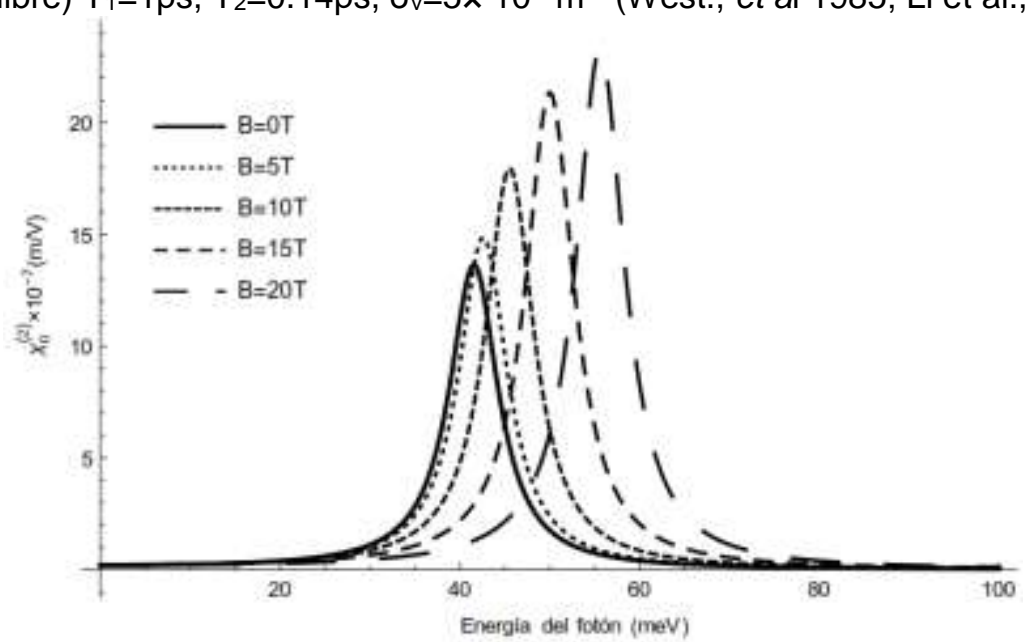

Fig. 1: Coeficiente de OR como una función de la energía del fotón incidente $\hbar \omega$ para cinco valores diferentes del campo magnético externo $(B=0 T, B=5 T, B=10 T, B=15 T, B=20 T)$, con $L=10 \mathrm{~nm}, R=8 \mathrm{~nm}$.

El coeficiente de rectificación óptica $\chi_{0}^{(2)}$ como una función del fotón incidente, en un CQD con $L=10 \mathrm{~nm}$ y $R=8 \mathrm{~nm}$ se muestra en la Fig. 1, para cinco valores diferentes del campo magnético. Los estados cuánticos considerados en esta transición involucran estados que tienen diferente solución radial, esto permite 
apreciar fácilmente la influencia del campo magnético sobre la OR, puesto que las soluciones axiales no son afectadas por B como lo indican las Ecuaciones (10) y (11). Se observa en la Fig. 1 un desplazamiento del pico de resonancia de $\chi_{0}^{(2)}$ a regiones de energías altas a medida que aumenta $B$, esto indica que el aumento del confinamiento de los portadores de carga producido por el aumento del campo magnético genera un corrimiento al azul en las energías de transición de los electrones en QD's semiconductores. Esta propiedad es de mucha utilidad en aplicaciones de opto-electrónica.

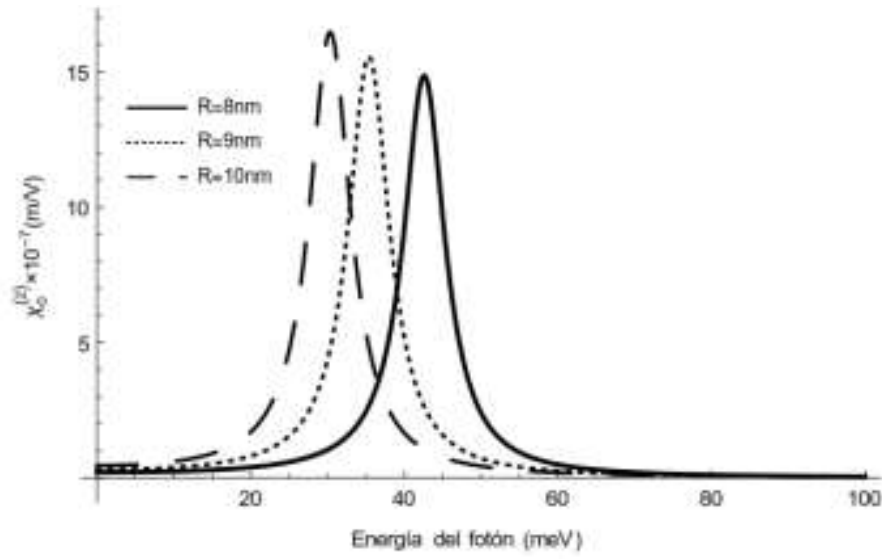

Fig. 2: Coeficiente de OR como una función de la energía del fotón incidente $\hbar \omega$ para tres valores diferentes del radio del $C Q D(R=8 \mathrm{~nm}, R=9 \mathrm{~nm}, R=10 \mathrm{~nm})$, con $L=10 \mathrm{~nm}, \mathrm{~B}=5 \mathrm{~T}$.

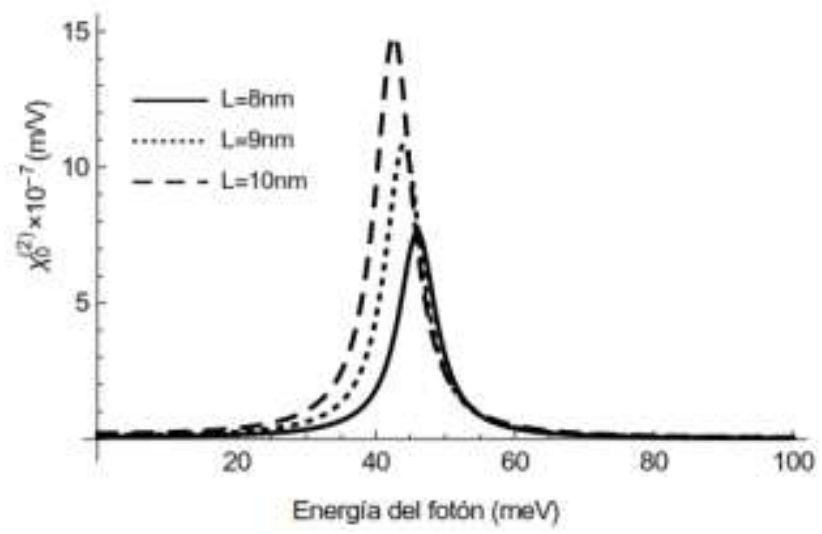

Fig. 3: Coeficiente de OR como una función de la energía del fotón incidente $\hbar \omega$ para tres valores diferentes de la longitud del $C Q D(L=8 n m, L=9 n m, ~ L=10 n m)$, con $L=8 \mathrm{~nm}, B=5 T$.

El efecto del tamaño del CQD sobre el coeficiente de OR se muestra en las figuras 2 y 3 . Se encontró que el aumento de las dimensiones del CQD: i) desplaza la posición del pico de resonancia en $\chi_{0}^{(2)}$ a regiones de bajas energías (corrimiento al rojo). Esto se debe a que al disminuir las dimensiones de una nano-estructura el confinamiento cuántico se hace más fuerte lo cual se refleja en un aumento de la separación de los niveles de energía. ii) La intensidad del pico de resonancia en $\chi_{0}^{(2)}$ aumenta. El origen físico radica en que el momento dipolar eléctrico de transición aumenta con el aumento de las dimensiones de las nanoestructuras y con el aumento del campo magnético (Wenfang., 2014).

\section{CONCLUSIONES}

Se presenta un estudio teórico sobre el efecto del campo magnético sobre la OR en un CQD de GaAs/A/GaAs que incluye dentro del QD potenciales parabólicos tanto en dirección radial como a lo largo de z (Khordad., 2013; Li et al., 2014) y un término asimétrico en la coordenada z un potencial sometido a un campo magnético uniforme. Además fuera del QD la nano-estructura presenta un potencial constante asociado a la matriz de $A / G a A s$. Los cálculos fueron realizados usando el formalismo de la matriz densidad dentro de la aproximación masa efectiva. Se encontró que el aumento en las dimensiones del CQD y/o la intensidad del campo magnético causan sobre el pico de resonancia un aumento en su intensidad y un desplazamiento hacia regiones de energías bajas (corrimiento al rojo). La razón física de este comportamiento se atribuye al efecto del confinamiento cuántico generado por la variación de la intensidad del campo magnético y de las dimensiones de la nano-estructura. 
En resumen, se encontró que la intensidad de campo magnético externo y el tamaño de las nanoestructuras juegan un papel importante en las propiedades ópticas no lineales que presentan los semiconductores de baja dimensionalidad, abriendo así nuevas oportunidades para la exploración práctica del efecto cuántico de tamaño y/o la acción de agentes externos en los dispositivos nano-estructurados.

\section{AGRADECIMIENTOS}

Los autores agradecen a la Universidad de los Llanos por el apoyo brindado para la realización de este trabajo enmarcado dentro del proyecto de investigación "Efecto del campo magnético sobre la respuesta óptica no lineal en un punto cuántico cilíndrico asimétrico - C03-F02-34-2015"

\section{REFERENCIAS}

Abdullah, M.; F.T. Mohammed y A.H. Al-Khursan, Second-order nonlinear susceptibility in quantum dot structure under applied electric field, doi:10.1016/j.spmi.2015.01.041, Superlattices and Microstructures: 82 (1), 219-233 (2015)

Akimoto, M. y otros cuatro autores, Effect of defects in TiO2 nanotube thin film on the photovoltaic properties of quantum dot-sensitized solar cells, doi:10.1016/j.tsf.2015.07.038, Thin Solid Films: 590(1) 90-97 (2015)

Duque, C.A. y otros cuatro autores, Intense laser effects on nonlinear optical absorption and optical rectification in single quantum wells under applied electric and magnetic field, doi:10.1016/j.apsusc.2010.09.095, Appl. Surf. Sci: 257(6) 2313-2319. (2011)

Hoyos, J.H. y otros tres autores, Nonlinear optical response in a zincblende GaN cylindrical quantum dot with donor impurity center, doi:10.1016/j.physb.2015.12.038, Physica B: 484 (1) 73-82 (2016)

Khordad, R., Effect of magnetic field on linear and nonlinear optical properties in a parabolic cylindrical quantum dot, doi: 10.1007/s12596-012-0104-y, J opt: 42(2), 83-91 (2013)

$\mathrm{Li}, \mathrm{X}$. y otros tres autores, Nonlinear optical rectification in asymmetric quantum dots with an external static magnetic field, doi:10.1016/j.physe.2013.08.025, Physica E: 56(1), 130-133 (2014)

Liu, G; G. Kangxian y W. Chao, Linear and nonlinear intersubband optical absorption in a disk-shaped quantum dot with a parabolic potential plus an inverse squared potential in a static magnetic field, doi:10.1016/j.physb.2012.03.030, Physica B: 407(12) 2334-2339 (2012)

Rezaei, G. y otros tres autores, Optical rectification coefficient of a two-dimensional parabolic quantum dot: Effects of hydrogenic impurity, external fields, hydrostatic pressure and temperature, doi:10.1016/j.physb.2014.06.008, Physica B: 451(1) 1-6 (2014)

Rosencher, E.; Ph. Bois, Model system for optical nonlinearities: Asymmetric quantum wells, doi:10.1103/PhysRevB.44.11315, Phys. Rev. B: 44 (20), 11 315-11 327 (1991)

Sakar S. y otros tres autores, Modulating nonlinear optical properties of impurity doped quantum dots via the interplay between anisotropy and Gaussian, doi:10.1016/j.spmi.2015.12.023, Superlattices and Microstructures: 90(1) 297-307(2016)

Shojaei, S. y A. Soltani, Nonlinear optical rectification of hydrogenic impurity in a disk-like parabolic quantum dot: The role of applied magnetic field, doi:10.1016/j.physe.2015.01.034, Physica E: 70(1) 108-112 (2015)

Vaseghi, B. y otros tres autores, Optical rectification and third harmonic generation of spherical quantum dots: Controlling via external factors, doi:10.1016/j.physb.2014.10.020, Physica B: 457(1) 212-217(2015)

Villamil, P., Donor in cylindrical quantum well wire under the action of an applied magnetic field, doi:10.1016/j.physe.2010.06.001, Physica E: 42 (9) 2436-2440 (2010)

Wang, G., Highly efficient third-harmonic generation from resonant intersubband transitions in core/shell spherical quantum dots, doi:10.1016/j.optcom.2015.06.045, Optics Communications: 355(1) 1-5 (2015)

Xie, W., Effect of an electric field on the nonlinear optical rectification of a quantum ring, doi:10.1016/j.physb.2014.03.003, Physica B: 443 (1) 60-62 (2014)

Zhang, Z.H. y otros cuatro autores, Nonlinear optical rectification in cubical quantum dots, doi:10.1016/j.physb.2009.04.035, Physica B: 404(16) 2332-2335 (2009)

West, E.; S. J. Eglash, First observation of an extremely largedipole infrared transition within the conduction band of a GaAs quantum well, doi:10.1063/1.95742, Appl. Phys. Lett: 46 (12), 1156-1158 (1985) 